\title{
O uso da tecnologia no ensino da anatomia humana: revisão sistemática da literatura de 2017 a 2020
}

\author{
The use of technology in human anatomy teaching: \\ a systematic review of the literature from 2017 to 2020

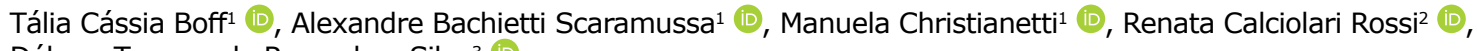 \\ Débora Tavares de Resende e Silva ${ }^{3}$ (D)
}

\section{RESUMO}

A tecnologia destaca-se como agente de avanço, principalmente na área da saúde. O estudo da anatomia humana nos cursos de medicina permanece tradicionalista com palestras teóricas e dissecção de cadáveres, por isso, metodologias tecnológicas parecem auxiliar o ensino, tornando-o interativo. Assim, o objetivo do estudo é analisar as perspectivas do uso de tecnologias no ensino da anatomia humana para inseri-las na graduação em medicina. Realizou-se uma revisão sistemática da literatura abrangendo artigos publicados entre janeiro de 2017 a março de 2020 nas bases de dados eletrônicas PubMed e Science Direct. Elencaram-se três critérios de inclusão: estudos com estudantes de medicina ou direcionado aos estudantes de medicina, estudos com residentes de medicina e estudos que avaliam o uso do desenvolvimento tecnológico no ensino da anatomia humana; e três de exclusão: estudos com animais, estudos com estudantes de outros cursos da área da saúde e estudos envolvendo área ou técnica cirúrgica específica. Onze artigos foram lidos na íntegra. Como resultado smartphones, código de resposta rápida (QR), realidade virtual (VR), modalidades impressas tridimensionais (3DP), próteses em 3D e outras tecnologias beneficiaram os alunos no aprendizado anatômico, os quais relataram instrumento atraente, de fácil manejo e acessível mesmo distante do laboratório. Além disso, demonstrou-se maior interesse dos acadêmicos em associar métodos tradicionais com as novas tecnologias. As tecnologias se mostraram eficazes no ensino da anatomia humana, visto que a maior parte dos estudos comprovou seu potencial enriquecedor nas avaliações que atingiram resultados iguais e melhores do que o ensino com as práticas tradicionais.

Palavras-chave: Anatomia; Educação Médica; Desenvolvimento Tecnológico.

\begin{abstract}
Technology stands out as an advancing agent, especially in the area of health. The study of human anatomy in medical courses remains traditionalist with theoretical lectures and dissection of cadavers, so technological methodologies seem to assist the teaching, making it interactive. Thus, the objective of the study is to analyze the perspectives of the use of technologies in the teaching of the human anatomy to insert them in undergraduate medical courses. A systematic review of the literature was carried out covering papers published between January 2017 and March 2020 in the electronic databases PubMed and Science Direct. Three inclusion criteria were listed: studies with medical students or targeted to medical students, studies with medical residents, and studies that evaluate the use of technological development in the teaching of human anatomy; and three exclusion criteria: studies with animals, studies with students from other health courses, and studies involving specific surgical area or technique. Eleven papers were read in full. As a result, smartphones, rapid response code (QR), virtual reality (VR), three-dimensional printed modalities (3DP), 3D prostheses, and other technologies benefited the students in anatomical learning, who reported an attractive instrument, easy to handle, and accessible even far from the laboratory. Technologies have proven to be effective in teaching human anatomy, given that most studies have proven their enriching potential in assessments that have achieved similar and better results than teaching with traditional practices.
\end{abstract}

Keywords: Anatomy; Medical Education; Technological Development.

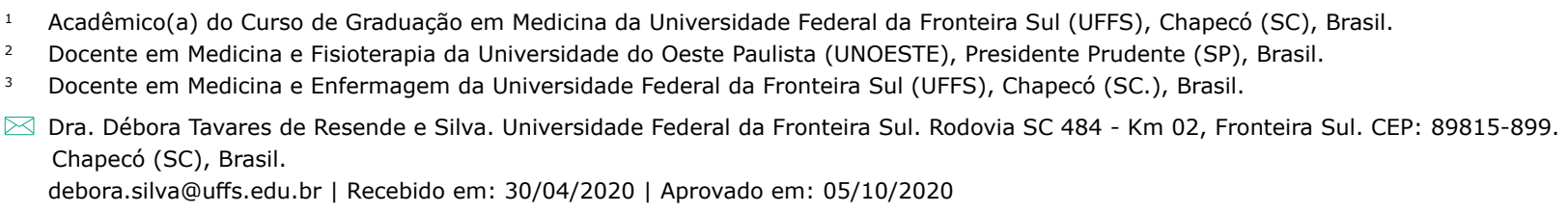




\section{INTRODUÇÃO}

O ensino da anatomia humana é historicamente uma pedra angular dentro da educação médi$\mathrm{ca}$, independentemente de nação ou especialidade, sendo as dissecações e palestras didáticas a pedagogia predominante antes da revolução tecnológica. Além disso, é uma área ampla e que necessita ser revisada e examinada de maneira frequente devido sua relação direta com o ambiente clínico e cirúrgico ${ }^{1}$.

A exposição dos estudantes de medicina desde o início do curso ao cadáver foi à essência do aprendizado anatômico desde o renascimento, visto que o processo de dissecção reforça o conhecimento adquirido em aulas teóricas e possibilita tocar e reconhecer os diferentes tecidos do organismo, sua consistência, formato e textura. Essa técnica de aprendizado ainda é vista como promotora de valores humanísticos, em que os estudantes, na maioria das vezes, têm o primeiro contato com a morte ${ }^{2,3}$.

Sabe-se que o uso cadavérico ainda é um dos métodos mais eficazes no ensino da anatomia humana, porém desde a Terceira Revolução Industrial, iniciada no século passado, ocorreu o crescimento e a expansão da tecnologia digital, a qual passou a ser inserida dentro das disciplinas de anatomia. Assim, houve mudanças de uma abordagem convencional mais centralizada para uma integralidade de métodos de ensino diferenciados. Isso segue o relatório de 2001 da Associação Americana de Faculdades de Medicina (AAMC), o qual destacou que o ensino anatômico deve incluir não apenas fatos e princípios aplicáveis aos seres humanos, mas também, dar ênfase aos aspectos comportamentais e sociais da saúde e doença, os quais podem ser alcançados com o uso de tecnologias diferentes que unam a anatomia com casos clínicos reais, perguntas e respostas, entre outros ${ }^{4,5}$.

Uma mudança drástica ao longo dos anos na graduação em medicina foi a redução da carga horária destinada a anatomia, que sofreu redução para a inserção de disciplinas integrativas com intuito de formar um profissional dotado de técnicas diferenciadas, desde as tradicionais até as mais inovadoras. Para isso, módulos especiais de estudo, oficinas baseadas em problemas, uso das redes sociais, aplicativos e programas 3D começaram a ser utilizados com o objetivo de otimizar o tempo reduzido e possibilitar aos estudantes 0 acesso até mesmo em casa de uma anatomia mais realista que a contida em livros didáticos. Além disso, novas metodologias contribuem para aquisição de um conhecimento aprofundado das estruturas humanas juntamente com a capacidade de aplicar diante da clínica médica ${ }^{6}$.

Com isso, o presente estudo de revisão sistemática da literatura tem por objetivo avaliar o uso das tecnologias no ensino da anatomia humana. Nesse sentido, busca-se avaliar se há uma discrepância considerável entre o ambiente de estudos presencial (em laboratório com uso de peças e cadáveres) e o estudo através do ambiente tecnológico (com o uso de tecnologias como aplicativos, robôs e outros). A partir disso, visa-se analisar se há perspectivas de há maior qualidade no aprendizado da anatomia com a inserção das tecnologias no ambiente convencional dos laboratórios.

\section{METODOLOGIA}

O presente estudo se trata de uma revisão sistemática da literatura, portanto não necessita aprovação ética, sendo realizada no período de janeiro de 2017 a março de 2020 a partir de duas bases de dados eletrônicas: PubMed e Science Direct. Os descritores utilizados na busca foram "anatomy" AND "medical education" AND "technological development". Apresenta-se o fluxograma na Figura 1 para simplificar de modo geral a seleção dos artigos. Sobretudo, o estudo foi dividido em cinco etapas: (1) formulação da questão, (2) localização dos estudos, (3) avaliação e seleção dos estudos, (4) análise e síntese, e (5) descrição dos resultados. Para tanto, elencou-se critérios de inclusão: 1) estudos com estudantes de medicina ou direcionado aos estudantes de medicina, 2) estudos com residentes de medicina e 3 ) estudos que avaliam o uso do desenvolvimento tecnológico no ensino da anatomia humana; além de critérios de exclusão: 1) estudos com animais, 2) estudos com estudantes de outros cursos da área da saúde e 3) estudos envolvendo área cirúrgica ou técnica cirúrgica. A seleção dos estudos ocorreu após a leitura e análise dos títulos, 
dos resumos e dos textos integrais por três pesquisadores independentes (TCB; ABS; MC; ), com amparo de um quarto pesquisador, sênior, para leitura e revisão da escrita (DTRS). A coleta de dados e informações foram divididas em três domínios: I) quais tecnologias foram desenvolvidas para o estudo da anatomia humana; II) qual a percepção dos estudantes de medicina perante o uso da tecnologia no aprendizado e III) quais são os resultados da utilização de metodologias ativas com o uso da tecnologia no ensino da anatomia humana. Por fim, devido ao caráter descritivo do presente estudo não foi avaliado risco de viés/qualidade metodológica de cada estudo.

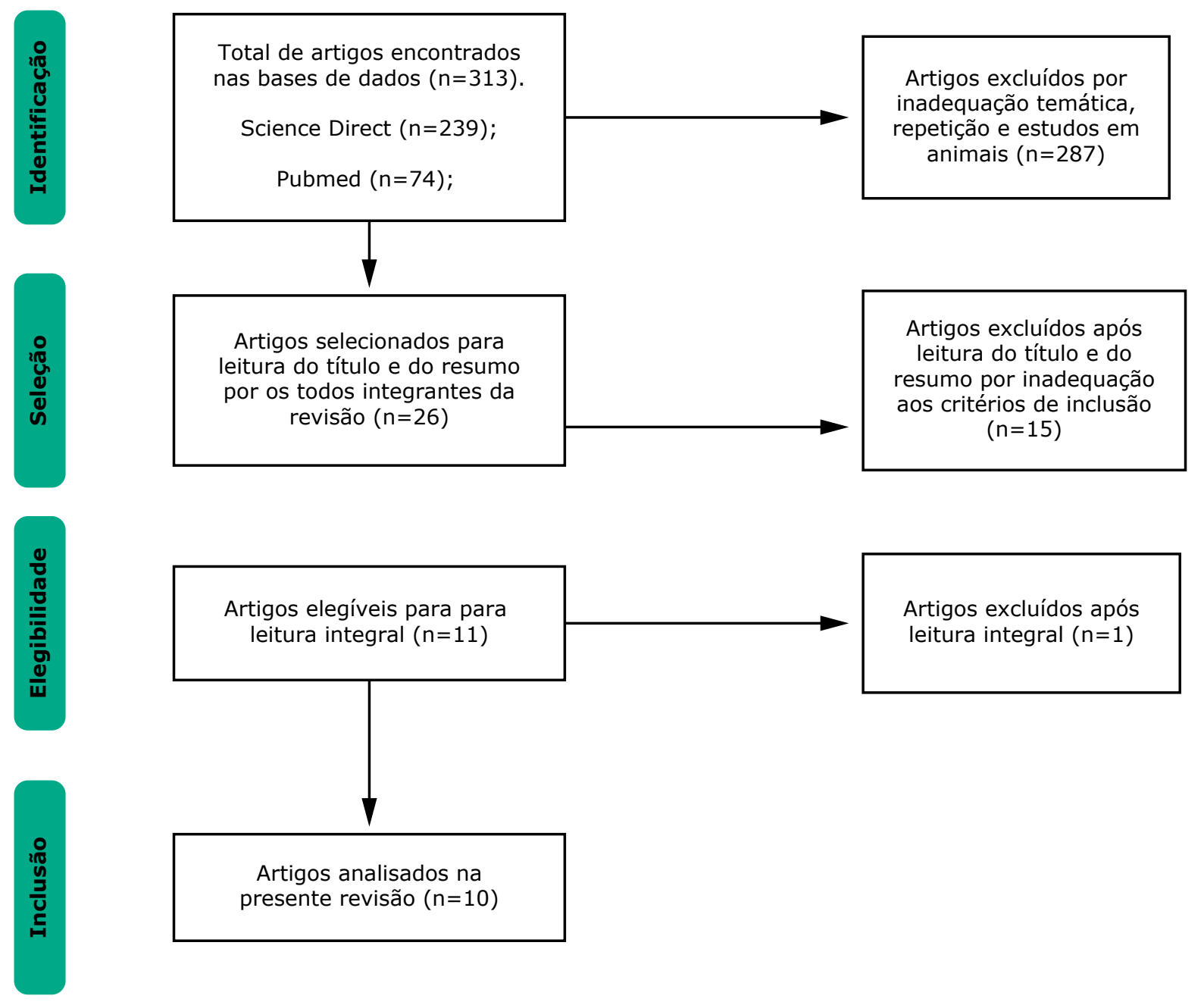

Figura 1. Organograma referente ao processo de seleção dos artigos para a revisão sistemática da literatura.

\section{RESULTADOS}

Primeiramente, identificaram-se no total 313 artigos ( 74 da base de dados PubMed e 239 na base de dados Science Direct). Após, devido inadequação à temática proposta, repetição de artigos e também pelo uso em animais, descartaram-se 287 artigos.
Assim, reuniram-se 26 artigos para todos os integrantes do grupo realizarem a leitura dos títulos e dos resumos. Com isso, 15 artigos foram excluídos, pois avaliavam o uso da tecnologia em técnicas cirúrgicas e com os residentes de medicina, ou seja, estavam inadequados aos critérios de inclusão. Em suma, 11 artigos foram lidos na íntegra por 
todos os revisores do grupo e excluí-se um artigo devido não correlacionar o estudo com o ensino da anatomia a estudantes da medicina ou profissionais da saúde. Assim, dos 11 artigos lidos, 10 atenderam aos critérios de elegibilidade para a revisão e estão apresentados de modo geral na Tabela 1.

Foi realizado um estudo com estudantes de medicina do primeiro ano submetidos a tutorial em vídeo guiado com imagens cirúrgicas laparoscópicas para o aprendizado da anatomia de estruturas do saco menor e da junção gastroesofágica. Como resultado, houve uma resposta positiva sobre o interesse e a satisfação do aluno em pré e pós-teste. A pontuação média dos alunos melhorou de 39\% para $88 \%$ após o vídeo. Os alunos alegaram que os vídeos ajudaram a aprender a anatomia da junção gastroesofágica e do saco inferior sendo uma adição valiosa à dissecção de cadáveres embalsamados ${ }^{7}$.

$\mathrm{Na}$ Índia um estudo avaliou o uso do smartphone como ferramenta educacional direcionada aos estudantes de medicina. Aproximadamente $96 \%$ da população estudada possuía smartphone e as aplicações mais utilizadas foram Dicionário de Anatomia e Medicina pelos alunos do primeiro ano, Dicionário Médico, Medscape e Google/Wikipedia no segundo e Medscape, Google/Wikipedia e Prognóstico/Diagnóstico no terceiro. Os dados do estudo revelaram que a maioria dos estudantes tem habilidades para fazer o uso como ferramenta digital de estudo, em que $37,2 \%$ já utilizam os smartphones para fins clínicos, além de $79,4 \%$ que são favoráveis à introdução dessa ferramenta no curso. Vale ressaltar que o estudo trouxe algumas contrapartidas como o uso inadequado pelo excesso de horas diárias gerando dependência, bem como distúrbios do sono, cefaleia e cansaço visual ${ }^{8}$.

Um estudo analisou o uso da realidade virtual para o campo da anatomia humana como ferramenta na educação médica. Para isso, desenvolveu-se um software executado com o uso de óculos estereoscópicos, em que os usuários têm percepção de estar dentro do ambiente virtual neste estudo, composto pelas estruturas do crânio, as quais podem ser vistas detalhadamente tanto internamente quanto externamente, além de acompanhadas por explicações em áudio. Dessa forma, a presente tecnologia foi atraente para os estudantes, pois possibilitou a inserção mais aprofundada no conteúdo, que contribuiu para visualização de estruturas que, muitas vezes, são difíceis de imaginar em imagens ou peças cadavéricas ${ }^{9}$.

Outro estudo investigou a importância do uso de modelos impressos tridimensionais (3DP) como forma de substituir ou aprimorar os recursos metodológicos existentes no estudo da anatomia humana. Para isso, criou-se um 3DP do membro superior de modo multicolorido e com multimateriais com a demonstração de músculos, nervos, artérias e ossos com resolução espacial de $1 \mathrm{~mm}$. Após, buscou-se examinar o valor educacional perante 0 aluno. Os alunos consideraram as características anatômicas nos modelos 3DP precisas com destaque para o código de cores por tecido, flexibilidade e pela necessidade de menor cuidado no manuseio e no exame da amostra e isso facilitou a apreciação das estruturas anatômicas. Em contrapartida, os estudantes relataram que as características clínicas dos modelos 3DP são menos realistas em comparação com as amostras plastinadas, por isso $53 \%$ ainda consideram as próteses plastinadas superiores. Mas, $87 \%$ acreditam que a combinação entre modelos 3DP e próteses plastinadas sejam ainda mais eficazes no aprendizado anatômico ${ }^{10}$.

Um estudo objetivou comparar a eficiência de um software 3D chamado BioDigital Human e próteses cadavéricas em laboratório, sendo o estudo realizado entre dois grupos de graduação compostos por estudantes de medicina do primeiro ano sem conhecimentos anatômicos prévios. Os alunos foram submetidos a um exame com imagens cadavéricas e 3D após o processo educacional, além de serem avaliadas as percepções discentes. Os resultados mostraram que os alunos que utilizaram o software 3D apresentaram melhor desempenho nos exames, comparados aos estudantes em uso de prótese. Em relação às percepções do uso dessa tecnologia, não foi encontrada diferença estatisticamente significante em relação à satisfação dos alunos sobre o uso de cada um dos métodos de aprendizagem. Ademais, dos que fizeram uso da tecnologia 3D, $72 \%$ afirmaram estarem satisfeitos ou muito satisfeitos com a metodologia ${ }^{11}$. 
Outro estudo abordou as diferentes atividades de aprendizagem alternativas de ensino (TLAs) com o objetivo de descobrir sua influência perante a percepção dos alunos nos exames de anatomia musculoesquelética. Alunos do primeiro e segundo ano de medicina de uma mesma escola médica foram comparados por um exame, em que um grupo completou 15 horas em dissecção cadavérica e o outro foi exposto a TLAs como próteses cadavéricas. Os dados suportam o ensino com cadáveres, imagens e cenários clínicos baseados em casos como elementos-chave para o currículo de anatomia. Além disso, os dados informam que as palestras didáticas e de aprendizado baseado em computador foram os menos eficazes para atingir os objetivos de ensino. O destaque aos casos clínicos se deve a ênfase de causar um ambiente centrado no aluno que busca informações clínicas e básicas de anatomia, patologia e ainda os integra para gerar um raciocínio clínico ${ }^{12}$.

Uma plataforma inovadora de aprendizado móvel foi desenvolvida com a utilização de códigos de resposta rápida $(\mathrm{QR})$ para os espécimes do museu Kong Chain School of Medicine, em que os QR foram vinculados a documentos em PDF com anotações, patologia e histórico clínico conforme os espécimes vistos. Os testes foram feitos com alunos do segundo ano de medicina sobre o sistema gastrointestinal. A maioria dos estudantes concordou sobre o fácil acesso às informações sobre a amostra com códigos QR, 96\% dos estudantes se disseram capazes de correlacionar o espécime com as imagens anotadas e $78 \%$ dos estudantes afirmaram que os códigos são úteis para a aprendizagem ${ }^{13}$.

Outro estudo, também buscou estimar o efeito de um modelo anatômico de realidade virtual (RV) chamado VisCubeSXTM sobre o conhecimento da anatomia do assoalho pélvico feminino de 32 residentes separando-os entre um grupo em RV e outro nos métodos tradicionais. Como resultado, houve melhora significativa nas pontuações pré e pós-teste tanto nas práticas tradicionais quanto no VisCubeSX $\mathrm{Tm}$. Os residentes expostos ao modelo anatômico VisCubeSX ${ }^{\mathrm{T} M}$ relataram que concordam sobre a contribuição do modelo para o aprendizado e que este melhora o atendimento ao paciente. Por fim, os residentes reportaram que eles gostaram do programa e que usariam se tivessem acesso ${ }^{14}$.

Outro modelo em 3D, dessa vez da coxa, permite o movimento em tempo real do modelo, a dissecção virtual pela remoção de componentes anatômicos, por exemplo, músculo reto femoral (RF). Esse é um dos primeiros estudos a detalhar o desenvolvimento e o uso de um modelo de retalho virtual 3D no currículo de graduação em medicina com destaque a preferência impressionante do usuário por esse modelo de retalho em relação aos métodos tradicionais de ensino, em que três quartos dos estudantes consideraram o modelo 3D como primeira escoIha. Os estudantes também relataram uma preferência pelo modelo virtual pela possibilidade de refazer a dissecção diversas vezes, ao contrário da dissecção em cadáveres que só é realizada, limitadamente, uma única vez ${ }^{15}$.

Um estudo com Realidade Virtual Interativa (URA) foi realizado com três grupos aleatórios de estudantes de medicina que estudaram a anatomia do coração humano com três métodos diferentes de aprendizado - um trabalho (texto e imagens), um 3D interativo de coração humano apresentado em uma tela de computador e um modelo de coração humano de URA. Antes e depois de cada sessão de aprendizagem os alunos realizaram o teste de anatomia do coração humano com 28 perguntas abertas. A partir do número de respostas corretas do teste de conhecimento avaliou-se o efeito de várias formas de material didático e sua eficiência que resultou em dois resultados principais: em primeiro lugar, o uso de URA no grupo experimental produziu um aumento significativo no conhecimento em comparação com os outros dois grupos. Em segundo lugar, o aumento do número de respostas corretas no grupo URA foi maior nos indivíduos com nível mais baixo de conhecimento na sessão de pré-aprendizagem. Estudar no ambiente de URA mostrou-se mais eficiente do que ler textos ou interagir com um modelo 3D na tela do computador ${ }^{16}$. 


\section{Tabela 1}

Síntese geral dos artigos analisados.

\begin{tabular}{|c|c|}
\hline Primeiro autor / Ano & Apresentação do estudo \\
\hline Dylan Isaacson / 2017 & $\begin{array}{l}\text { Utilizou um conteúdo multimídia de uma sessão de laparoscopia realizada em } \\
\text { cadáveres frescos com foco na anatomia abdominal, destinado a estudantes de } \\
\text { medicina do primeiro ano. Assim, avaliou o impacto dos vídeos de laparoscopia } \\
\text { antes dos alunos obterem o primeiro contato com a anatomia abdominal em cadá- } \\
\text { veres no laboratório de anatomia. }\end{array}$ \\
\hline
\end{tabular}

Monika Y Gavali / 2017

Santiago González Izard / 2017

Sreenivasulu Reddy Mogali / 2018

Vasileios Mitrousias / 2018

Jason Peeler / 2018

Sreenivasulu Reddy Mogali / 2019

David RichEllington / 2019

StevenLo / 2020

Y.P.Zinchenko / 2020
Avaliou o uso de smartphones como ferramenta educacional entre os estudantes de medicina do primeiro ao terceiro ano, e além disso, buscou identificar sua aplicação médica.

Apresentou um Software de Realidade Virtual (executado em óculos estereoscópicos e acompanhado com explicações em áudio e texto), além do uso do celular para a realização de movimentos com as mãos. Ilustrou o potencial de ensino da aplicação da realidade virtual no campo da anatomia humana.

Uso de um modelo impresso tridimensional (3DP) multicolorido e de multimaterial do membro superior com base em uma prótese plastinada, capturando músculos, nervos, artérias e ossos com uma resolução espacial de $\pm 1 \mathrm{~mm}$. Examinou o valor educacional do modelo 3DP comparado ao plastinado diante de alunos do segundo ano de Medicina.

Uso de um modelo de software BioDigital Human em 3D. Investigou qual método é associado com melhor resultado, comparando a performance nos exames dos alunos, quando comparados entre o aprendizado em leituras e cadáveres com o software em 3D.

Uso do Ensino Alternativo e Atividades de Aprendizagem (TLAs), a partir disso, examinou retrospectivamente como os diferentes TLAs influenciam a aprendizagem e desempenho dos alunos no que tange o ensino da anatomia musculoesquelética.

Estudo utilizou dos Códigos de resposta rápida $(\mathrm{QR})$ para verificar a eficácia como uma ferramenta de aprendizado com 120 estudantes de medicina do segundo ano no ensino sistema gastrointestinal.

Fez o uso do Sistema VisCubeSX ${ }^{\text {TM }}$, um simulador de realidade virtual, e a partir deste estimou a efetividade da adição da Realidade Virtual anatômica do assoalho pélvico feminino no ensino dos residentes, quando comparado com o estudo tradicional.

Avaliou o uso do modelo 3D Anterolateral da Coxa para fornecer um panorama amplo do processo de implementação de um modelo 3D na graduação médica.

Utilizou da realidade virtual imersiva (Sharecare) para identificar se os estudos com essa sistemática virtual se sobressaem ao uso de métodos tradicionais de ensino.

\section{DISCUSSÃO}

A educação em anatomia é fundamental para a Medicina é um rito de passagem para médicos alunos. Diferentemente das gerações anteriores, os alunos do século XXI exigem conectividade, ressonância e inovação em sua educação. A interação médico-paciente, o treinamento cirúrgico, a pesquisa e a educação científica estão interligadas a recursos em 3D, porém há limitações quanto aos custos e a complexidade para aquisição de equipamentos especializados. Os estudos mostram que ferramentas como a
Tomografia Computadorizada (TC) e a Ressonância Magnética (RM) são usadas para fabricar protótipos para geração de objetos 3D. Como exemplo, a Família Virtual composta por homem de 34 anos, mulher de 26 anos e dois filhos (menina de 11 anos e menino de 6 anos) baseados em alta resolução de RM, em que mais de 80 tecidos foram distinguidos durante a segmentação que subsequente foram reconstruídos como objetos de superfície produzindo imagens com alta precisão ${ }^{17,18}$.

Atualmente, a maioria do ensino sobre anatomia de superfície e a educação morfológica de 
procedimentos cirúrgicos é ensinada por simulação, sendo um exemplo a Visible Dataset Humano que demonstra um corpo inteiro que pode ser reconstruído volumetricamente pelo computador. Isso o torna um recurso para aplicações de anatomia humana em educação, modelagem, simulação, treinamento, morfometria e entretenimento mental ${ }^{19}$.

Outro equipamento adquirido por universidades foi a mesa anatômica virtual 3D como recurso complementar ao aprendizado da anatomia por estudantes de graduação em Medicina. Os alunos referiram que a mesa anatômica 3D virtual forneceu mais conhecimento de anatomia em relação ao conhecimento prévio estudando anteriormente em peças reais. Por isso, a mesa tem potencial para melhorar tanto a compreensão quanto o interesse dos estudantes pela anatomia ${ }^{20}$.

Dados sobre a aprendizagem por computador demonstrou que houve uma melhora dependente da dose, ou seja, quanto maior a quantidade de treinamento através dessa tecnologia, maior foi o desempenho das habilidades espaciais pelos estudantes ${ }^{21}$.

Da mesma forma, as estratégias modernas na educação médica visam o envolvimento dos alunos na aprendizagem e no treinamento para solucionar problemas. A participação dos alunos em grupos de trabalho propostas pelas técnicas mais modernas torna-se importante para a interpretação das patologias, pois thes permite aprender e interpretar imagens e facilitar a exploração física para os futuros pacientes como o projeto "O Humano Visível" que possibilita analisar diferentes estruturas do corpo humano22.

A essencialidade de novas modalidades para o uso da anatomia encontra-se na redução do tempo dedicado ao ensino da anatomia como resultado do aumento no número de alunos, bem como o aumento em áreas do curso e de conteúdos. Além disso, o aparecimento de novas e valiosas tecnologias na educação médica parecerá muito enriquecedor ${ }^{22,23}$.

Porém, mesmo com diversas tecnologias desenvolvidas, ainda permanecem argumentos referentes à importância da dissecção de cadáveres devido à primazia do paciente, apreensão do corpo multidimensional, percepção mediada pelo toque no cadáver, aprender a linguagem básica da medicina, competência em diagnóstico por imagem, aprendizagem em grupo de pares, treinamento para especialidades médicas. Assim, estudos mostram que quando conectado a dissecação, a informática médica pode agilizar e aprimorar a preparação para uma profissão médica baseada no paciente ${ }^{24}$.

Atualmente, devido às mudanças sociais, acredita-se que há um grande desafio para as universidades formarem um profissional de saúde com perfil criativo diante dos problemas do cotidiano. A utilização de cadáveres humanos é, ainda, considerada indispensável no processo de ensino-aprendizagem da anatomia humana, visto que o manuseio do cadáver possibilita fortalecer a humanização dos futuros profissionais da saúde. Dentro disso, excluir a prática com cadáveres gera incertezas, pois lacunas podem ser geradas pela ausência do contato com o corpo real. Por isso, é importante que o profissional formado conheça a tecnologia e entre em contato com ela, para ampliar seu repertório de informações em um ambiente amigável, melhorar a compreensão e a visualização das estruturas anatômicas e transformar o processo educacional mais eficiente e dinâmico ${ }^{25}$.

Para tanto, sabe-se que muitos educadores buscam novas alternativas com intuito de proporcionar um aprendizado eficiente aos discentes e que seja coerente com as mudanças da sociedade. Além disso, alguns vieses foram analisados como o custo para a aplicação da tecnologia que pode ser baixo como com o uso de um aplicativo para smartphone ou elevado com a aquisição de próteses em 3D ou, por exemplo, uma mesa anatômica e a preferência ainda marcante por peças cadavéricas. Portanto, deve-se analisar a realidade de cada universidade e assim reconhecer a possibilidade de adicionar a tecnologia no ambiente convencional, ou seja, laboratorial de aprendizado da anatomia humana, visto que essa associação apresenta o maior grau de satisfação por parte dos estudantes.

\section{CONCLUSÃO}

O artigo possibilitou concluir que há uma procura acirrada pelo desenvolvimento tecnológico, o qual já está inserido em áreas da saúde como pesquisas para desenvolver próteses, medicamentos, vacinas, além de técnicas aprimoradas de cirurgia como a laparoscopia e outros. Por isso, vale destacar a importância de instigar discussões e reflexões sobre a eficácia do uso da tecnologia associada ao 
ensino da anatomia humana nos cursos de graduação em medicina. Nesse sentido, a inserção de metodologias que fazem o uso da tecnologia associada ao ambiente tradicional dos laboratórios de anatomia se apresenta, atualmente, como um dos métodos mais eficazes para o aprendizado dos estudantes, visto que a maior parte dos estudos comprovou seu potencial enriquecedor nas avaliações que atingiram resultados iguais e melhores do que o ensino apenas com as práticas tradicionais.

\section{REFERÊNCIAS}

1. Sugand $\mathrm{K}$, Abrahams $\mathrm{P}$, Khurana A. The anatomy of anatomy: a review for its modernization. Anatomical sciences education. 2010; 3(2), 83-93.

2. McLachlan JC, Bligh J, Bradley P, Searle J. Teaching anatomy without cadavers. Medical education. $2004 ; 38(4), 418-424$.

3. Older J. Anatomy: a must for teaching the next generation. The Surgeon. 2004; 2(2), 79-90.

4. Drake RL, McBride JM, Lachman N, Pawlina W. Medical education in the anatomical sciences: The winds of change continue to blow. Anatomical sciences education. 2009; 2(6), 253-9.

5. Turney BW. Anatomy in a modern medical curriculum. The Annals of The Royal College of Surgeons of England. 2007; 89(2), 104-7.

6. AbouHashem Y, Dayal M, Savanah S, Štrkalj G. The application of 3D printing in anatomy education. Medical education online. 2015; 20(1), 29847.

7. Isaacson D, Green C, Topp K, O'Sullivan P, Kim E. Guided laparoscopic video tutorials for medical student instruction in abdominal anatomy. MedEdPORTAL: the journal of teaching and learning resources. 2017; 13. Disponível em: https://www.ncbi.nlm.nih.gov/pmc/ articles/PMC6342516/.

8. Gavali, MY, Khismatrao, DS, Gavali, YV, Patil, KB. Smartphone, the new learning aid amongst medical students. Journal of clinical and diagnostic research: JCDR. 2017; 11(5), JC05.

9. Izard SG, Méndez JAJ, Palomera PR. Virtual reality educational tool for human anatomy. Journal of medical systems. 2017; 41(5), 76.

10. Mogali SR, Yeong WY, Tan HKJ, Tan GJS, Abrahams PH, Zary N, Ferenczi, MA. Evaluation by medical students of the educational value of multi-material and multi-colored three-dimensional printed models of the upper limb for anatomical education. Anatomical sciences education. $2018 ; 11(1)$, 54-64.
11. Mitrousias $\mathrm{V}$, Varitimidis $\mathrm{SE}$, Hantes $\mathrm{ME}$, Malizos $\mathrm{KN}$, Arvanitis $\mathrm{DL}, \mathrm{Zibis} A H$. Anatomy learning from prosected cadaveric specimens versus three-dimensional software: A comparative study of upper limb anatomy. Annals of Anatomy-Anatomischer Anzeiger. 2018; 218, 156-164.

12. Peeler J, Bergen $\mathrm{H}$, Bulow A. Musculoskeletal anatomy education: Evaluating the influence of different teaching and learning activities on medical students perception and academic performance. Annals of AnatomyAnatomischer Anzeiger. 2018; 219, 44-50.

13. Mogali SR, Vallabhajosyula R, Hon Ng C, Lim D, Ang ET, Abrahams P. Scan and Learn: Quick Response Code Enabled Museum for Mobile Learning of Anatomy and Pathology. Anatomical sciences education. 2019; 12(6), 664-672.

14. Ellington DR, Shum PC, Dennis EA, Willis HL, Szychowski JM, Richter HE. Female pelvic floor immersive simulation: a randomized trial to test the effectiveness of a virtual reality anatomic model on resident knowledge of female pelvic anatomy. Journal of minimally invasive gynecology. 2019; 26(5), 897-901.

15. Lo S, Abaker ASS, Quondamatteo F, Clancy J, Rea P, Marriott $M$, et al. Use of a virtual 3D anterolateral thigh model in medical education: Augmentation and not replacement of traditional teaching? Journal of Plastic, Reconstructive \& Aesthetic Surgery. 2020; 73(2), 269-275.

16. Zinchenko YP, Khoroshikh PP, Sergievich AA, Smirnov AS, Tumyalis AV, Kovalev, A I et al. Virtual reality is more efficient in learning human heart anatomy especially for subjects with low baseline knowledge. New Ideas in Psychology. 2020; 59, 100786.

17. Rengier $F$, Mehndiratta $A$, Von Tengg-Kobligk $H$, Zechmann CM, Unterhinninghofen $\mathrm{R}$, Kauczor $\mathrm{HU}$, et al. 3D printing based on imaging data: review of medical applications. International journal of computer assisted radiology and surgery. 2010; 5(4), 335-341.

18. Christ A, Kainz W, Hahn EG, Honegger K, Zefferer M, Neufeld $E$, et al. The Virtual Family-development of surface-based anatomical models of two adults and two children for dosimetric simulations. Physics in Medicine \& Biology. 2009; 55(2), N23.

19. Spitzer VM, Whitlock DG. The Visible Human Dataset: the anatomical platform for human simulation. The Anatomical Record: An Official Publication of the American Association of Anatomists. 1998; 253(2), 49-57.

20. Rosa BR, Correia MM, Zidde DH, Thuler LCS, Brito APCBD, Biolchini JCDA. Aprendizado da Anatomia Hepatobiliar pela Mesa Anatômica Virtual 3D. Revista Brasileira de Educação Médica. 2019; 43(1), 615-622.

21. Guimarães B, Firmino-Machado J, Tsisar S, Viana B, Pinto-Sousa M, Vieira-Marques $P$, et al. The role of 
anatomy computer-assisted learning on spatial abilities of medical students. Anatomical sciences education. 2019; 12(2), 138-153.

22. Vázquez R, Riesco JM, Juanes JA, Blanco $E$, Rubio $M$, Carretero J. Educational strategies applied to the teaching of anatomy. The evolution of resources. European Journal of Anatomy. 2007; 11(S1), 31-43.

23. Moro C, Štromberga Z, Raikos A, Stirling A. The effectiveness of virtual and augmented reality in health sciences and medical anatomy. Anatomical sciences education. 2017; 10(6), 549-559.

24. Aziz MA, Mckenzie JC, Wilson JS, Cowie RJ, Ayeni SA, Dunn BK. The human cadaver in the age of biomedical informatics. The Anatomical Record: An Official Publication of the American Association of Anatomists. 2002; 269(1), 20-32.

25. Costa GBFD, Lins CCDSA. O cadáver no ensino da anatomia humana: uma visão metodológica e bioética. Revista Brasileira de Educação Médica. 2012; 36(3), 369-373. 